\title{
Neutrophil Extracellular Traps in Arterial and Venous Thrombosis
}

\author{
Elodie Laridan, $\mathrm{MSc}^{1} \quad$ Kimberly Martinod, $\mathrm{PhD}^{1} \quad$ Simon F. De Meyer, $\mathrm{PhD}^{1}$ \\ ${ }^{1}$ Laboratory for Thrombosis Research, KU Leuven Campus Kulak \\ Kortrijk, Kortrijk, Belgium \\ Address for correspondence Simon De Meyer, PhD, Laboratory for \\ Thrombosis Research, KU Leuven Campus Kulak Kortrijk, E. Sabbelaan \\ 53, 8500 Kortrijk, Belgium (e-mail: simon.demeyer@kuleuven.be).
} Semin Thromb Hemost

\author{
Abstract \\ Keywords \\ - NETs \\ - thrombosis \\ - neutrophil \\ - stroke \\ - venous \\ thromboembolism \\ - myocardial infarction
}

Thrombotic complications are still a major health risk worldwide. Our view on the pathophysiology of thrombosis has significantly changed since the discovery of neutrophil extracellular traps (NETs) and their prothrombotic characteristics. Generated by neutrophils that release their decondensed chromatin as a network of extracellular fibers, NETs promote thrombus formation by serving as a scaffold that activates platelets and coagulation. The thrombogenic involvement of NETs has been described in various settings of thrombosis, including stroke, myocardial infarction, and deep vein thrombosis. The aim of this review is to summarize existing evidence showing the presence of NETs in human thrombus material. Following an introduction on NETs and their role in thrombus formation, the authors address studies showing the presence of NETs in arterial or venous thrombi. In addition, they focus on potential novel therapeutic opportunities to resolve or prevent thrombosis by targeting NETs.
In the last decade, neutrophil extracellular traps (NETs) have significantly changed our view on thrombosis. Thrombosis is caused by blood clots that hamper normal blood flow in arteries or veins, leading to several pathologies including ischemic heart disease, ischemic stroke, and venous thromboembolism (VTE). With one in four people dying worldwide from thrombotic conditions, thrombosis is a major contributor to global disease burden. ${ }^{1}$ To improve prevention, diagnosis, and treatment of thrombosis, a good understanding of its underlying mechanisms is essential. Interestingly, NETs have been identified as new DNA-based players in blood clot formation and thrombosis. Evidence of NETs being present in thrombi retrieved from thrombosis patients is growing and the impact of NETs in thrombosis is gaining increased attention. NETs have been found in different settings of thrombosis, including stroke, myocardial infarction, and deep vein thrombosis (DVT). ${ }^{2}$ In this review, we summarize current knowledge on the presence of NETs found in human thrombi. After introducing NETs and their role in thrombus formation, we address studies showing the presence of NETs in arterial or venous thrombi. We also discuss the potential clinical implications and novel therapeutic opportunities of targeting NETs in thrombosis.

\section{Neutrophil Extracellular Traps}

Neutrophil extracellular traps were first discovered as a novel immune defense mechanism of neutrophils. ${ }^{3}$ In 2004 , Brinkman et al described the ability of neutrophils to release decondensed chromatin that is decorated with granular proteins, forming a network of extracellular fibers. ${ }^{3}$ NETs create a physical barrier that prevents the spread of pathogens and facilitates killing microbes by high concentrations of antimicrobial proteins and phagocytosis by other phagocytes. ${ }^{3}$ Other than necrosis or apoptosis, NETosis is a well-orchestrated form of cell death during which neutrophils undergo important changes in their morphology after stimulation by a variety of agonists including pathogens, platelets, and noninfectious inflammatory stimuli. ${ }^{2,4,5}$ First, the nucleus loses its characteristic nuclear lobulation and subsequently swells (-Fig. 1). Second, due to swelling, the integrity of nuclear membrane as well as of granular membrane is lost, allowing mixing of
Issue Theme Editorial Compilation VI; Guest Editors: Emmanuel J. Favaloro, PhD, FFSc (RCPA), and Giuseppe Lippi, MD.
Copyright (c) by Thieme Medical Publishers, Inc., 333 Seventh Avenue, New York, NY 10001, USA.

Tel: +1(212) 584-4662.
DOI https://doi.org/ 10.1055/s-0038-1677040. ISSN 0094-6176. 


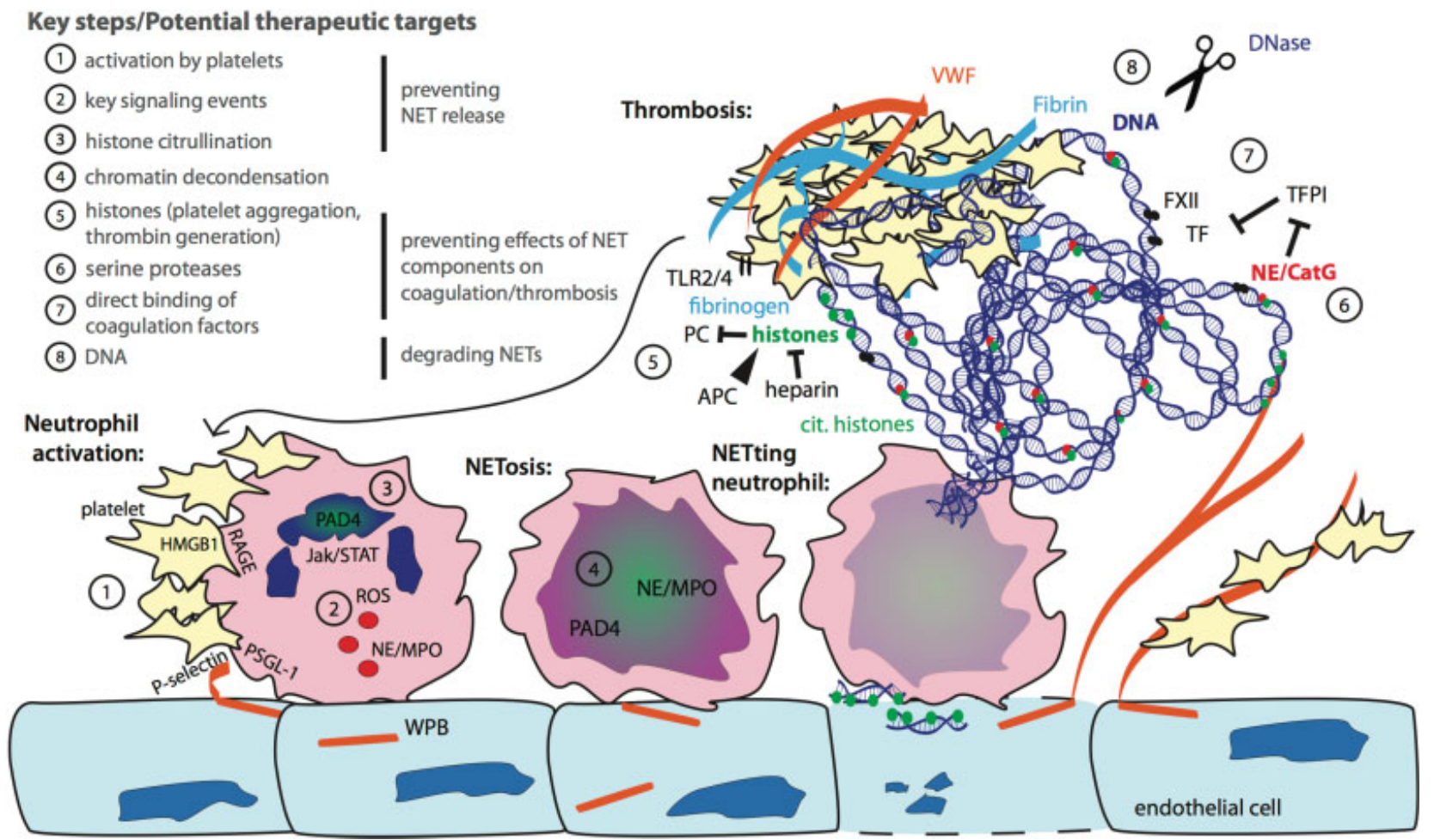

Fig. 1 Key steps in the NETosis pathway and interaction of NET components with prothrombotic factors provide insight into potential therapeutic targets. The process of NET formation in the context of thromboinflammation can be initiated by activation of the neutrophil by platelets, either mediated by HMGB1-RAGE or P-selectin-PSGL-1 interactions (1). Key intracellular neutrophil signaling occurs, including production of ROS and activation of the Jak-STAT pathway (2). Histone citrullination by PAD4 (3), which rapidly enters the nucleus, and nuclear translocation of NE and MPO drive chromatin decondensation and nuclear swelling accompanied by the loss of internal nuclear membranes (4). NETs are then released into the extracellular space, providing procoagulant/prothrombotic activity through various mechanisms linked to NET components. Histones (5) induce endothelial cell cytotoxicity, inhibit protein $\mathrm{C}$ activation promoting thrombin generation, and induce platelet aggregation either directly of via fibrinogen. Serine proteases inhibit TFPI (6), promoting tissue factor and factor XII-dependent coagulation and fibrin formation. These coagulation factors can also directly bind to NETs (7). NETs also form a scaffold for platelet adhesion, and VWF and fibrinogen binding, and thus targeting of thrombi with DNase (8) represents a promising therapeutic approach in thrombosis. APC, activated protein C; CatG, cathepsin G; HMGB1, high mobility group box 1 protein; Cit., citrullinated; FXII, factor XII; Jak/STAT, Janus kinase/signal transducer and activator of transcription proteins; MPO, myeloperoxidase; NE, neutrophil elastase; PAD4, peptidylarginine deiminase 4; PSGL-1, P-selectin glycoprotein ligand 1; RAGE, receptor for advanced glycation end products; ROS, reactive oxygen species; TF, tissue factor; TFPI, tissue factor pathway inhibitor; TLR2/4, toll-like receptors 2 and 4; VWF, von Willebrand factor; WPB, Weibel-Palade body.

chromatin with cytoplasmic and granular contents. Finally, the cytoplasmic membrane is compromised, resulting in release of NET into the extracellular space. Although the exact steps in NET formation are still poorly understood, some key players have been identified, including reactive oxygen species (ROS), ${ }^{5}$ granulocyte enzymes such as myeloperoxidase (MPO), and neutrophil elastase (NE), and involvement of enzyme peptidylarginine deiminase 4 (PAD4). A well-described aspect of NETosis is the citrullination of histones catalyzed by PAD4, a calcium-dependent enzyme which can enter the nucleus. PAD4 modifies arginine residues of histones $\mathrm{H} 3$ and $\mathrm{H} 4$ by citrullination, which causes a loss of positive charge, thus allowing decondensation of chromatin and hence swelling of the nucleus. The absence of PAD4 enzyme in mice prevents NET formation (NETosis) in isolated neutrophils, thus showing the importance of this enzyme in NETosis. ${ }^{6}$ Although ROS generated upon reduction of NAD phosphate (NADPH) oxidase have been described as a potential activator of PAD4 enzyme, the exact mechanism of how PAD4 is activated remains to be characterized. ${ }^{7}$ ROS are in turn responsible for release of MPO and NE from the azurophilic granules within the neutrophil. Besides their function as antimicrobial proteins, NE and MPO translocate to the nucleus to cleave histones and provide a synergizing effect, aiding the chromatin decondensation process in NETosis. ${ }^{8}$ NETs can be formed via different pathways, depending on the stimulus. Stimulation of neutrophils in vitro (e.g., with phorbol myristate acetate) induces the activation of NADPH oxidase complex and triggers NET formation in vitro. ${ }^{5}$ On the other hand, the calcium ionophore ionomycin induces NETosis by calciumdependent hyperactivation of PAD4. ${ }^{7}$ Additionally, certain types of bacteria can induce a distinct type of NET release called "vital NETosis."9

Interestingly, due to their ability to act as a scaffold, it has become clear that NETs, besides their role as immune strategy against pathogens, are also implicated in other disease settings like autoimmunity and thrombosis. 


\section{Neutrophil Extracellular Traps and Thrombosis}

The concept of immunothrombosis describes the interaction of the innate immune system and the activation of coagulation. The specific contribution of neutrophils in thrombus formation is only recently becoming better understood. Together with platelets, neutrophils are among the first cells recruited to sites of injury and/or infection. Neutrophils can limit the dissemination of microbial infections as part of their host defense mechanisms by promoting blood coagulation, for example, by enhancing fibrin deposition. ${ }^{10}$ This fibrin/NET network prevented bacterial invasion into surrounding tissue from the liver microvasculature, ${ }^{10}$ and disruption of the NET scaffold promoted systemic dissemination of bacteria. ${ }^{11}$ Dysregulation or excessive stimulation, in particular within the vasculature, can, however, lead to pathological thrombotic processes. ${ }^{12}$ Neutrophils thus regulate thrombosis via several mechanisms in which NETs play a central role.

One of the main ways by which NETs promote thrombosis is by forming a scaffold for adhesion of platelets, red blood cells (RBCs), and platelet adhesion molecules such as fibrinogen, von Willebrand factor (VWF), and fibronectin (-Fig. 1). ${ }^{13}$ This scaffold not only forms a structural basis but many of its components can also actively trigger platelet activation and blood coagulation. Histones $\mathrm{H} 3$ and $\mathrm{H} 4$ are highly cytotoxic to endothelial and epithelial cells, ${ }^{14}$ while they can also trigger platelet aggregation. ${ }^{13}$ Histones are able to interact with platelets via fibrinogen ${ }^{15}$ or directly via toll-like receptors (TLR) 2 and $4,{ }^{14}$ thus resulting in platelet activation and local increase of thrombin generation. ${ }^{16} \mathrm{By}$ binding to thrombomodulin, histones also prevent the activation of activated protein $\mathrm{C}$ (APC), thus boosting further thrombin generation. ${ }^{17}$ In addition, NETs promote both the intrinsic and extrinsic coagulation pathway, mainly through the activity of neutrophil serine proteases. NE and cathepsin $G$, which are also present on NETs, enhance tissue factor- and factor XII-driven coagulation via proteolysis of tissue factor pathway inhibitor (TFPI). ${ }^{10}$ NETs also directly bind factor XII and, with the cooperation of platelets, support its activation to factor XIIa. ${ }^{18}$ Interestingly, complete NET complexes do not have the same degree of procoagulant effect as their separate DNA and histone components, which individually have been shown to be more potent promoters of coagulation. ${ }^{19,20}$ Possibly, the tight packaging of histones and DNA into nucleosomes partly reduces the ability to interact with the coagulation system.

The interaction between NETs and platelets and, in turn, between activated platelets and neutrophils can stimulate the vicious circle leading to pathological thrombus formation. Indeed, activated platelets were shown to promote NETosis via pathways that are currently not completely elucidated, but most likely include P-selectin/P-selectin glycoprotein ligand 1 interaction, ${ }^{21}$ and release of damage-associated molecular patterns, such as highmobility group box 1 protein (HMGB1) $22-24$ and platelet TLRs. $^{25}$
Most of the evidence on NETs-mediated thrombosis is derived from animal studies or in vitro studies using single cell interactions. ${ }^{2}$ Whether or not NETs are directly involved in human pathological thrombus formation is less well studied. Yet, studies on retrieved human thrombus material have started to reveal that NETs are indeed important constituents of human thrombi. In the next sections, current knowledge regarding the presence of NETs in both arterial and venous thrombi retrieved from patients is discussed in more detail.

\section{Neutrophil Extracellular Traps in Arterial Thrombi}

Increasing evidence from studies on human thrombi indicates that NETs are part of arterial thrombi in various thrombotic pathologies, including myocardial infarction and ischemic stroke. Already before the discovery of NETs, it was shown that neutrophils were important constituents of culprit lesions causing acute myocardial infarction. ${ }^{26}$ More recent histological studies using novel specific markers revealed that NETs are also abundant throughout coronary thrombi retrieved from patients with acute myocardial infarction. ${ }^{22,27-29}$ A multicenter European study showed that neutrophils and NETs are also hallmarks of thrombi retrieved from patients with stent thrombosis after percutaneous coronary intervention. ${ }^{28}$ NETs in coronary thrombi were typically identified via histochemical and immunohistochemical stainings for extracellular DNA and histones, together with a neutrophil marker such as MPO or NE. NETs were found in close proximity of platelets, suggesting that activated platelets actively contribute to the incorporation of NETs in coronary thrombi, for example, via HMGB1. ${ }^{22}$ Serving as a scaffold for platelets, erythrocytes, and fibrin, NETs possibly contribute to thrombus growth and stabilization. Indeed, NETs were frequently observed in thrombi that were only a couple of days old, but not in older, more organized coronary thrombus specimens. ${ }^{27}$ Importantly, Mangold et al found that coronary thrombus NET burden correlated positively with infarct size and negatively with ST-segment resolution, indicating the potential clinical relevance of NETs in myocardial infarction. ${ }^{29}$ Also in ischemic stroke, NETs have been identified as an important constituent of occluding thrombi. We recently described the presence of NETs in 68 thrombi that were retrieved from ischemic stroke patients via endovascular thrombectomy (-Fig. 2). ${ }^{30}$ In these cerebral occlusions, neutrophils were abundant, and NETs were found in all thrombi. NETs were visualized and identified via staining for extracellular chromatin, NE, and citrullinated histone H3 (H3Cit). Since ischemic stroke can be caused by thrombi from different origins depending on stroke etiology, it is interesting to correlate thrombus composition with etiology. ${ }^{31}$ Although we did not find a correlation between presence of neutrophil and stroke etiology, other studies showed that stroke thrombi from cardioembolic origin are particularly rich in white blood cells. ${ }^{32,33}$ Accordingly, NETs were more abundant in stroke thrombi of cardioembolic origin compared 
H\&E

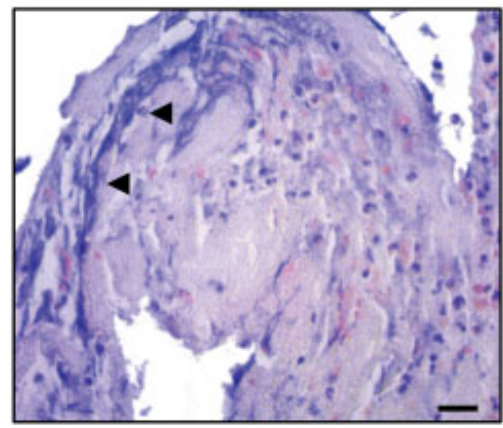

H3Cit

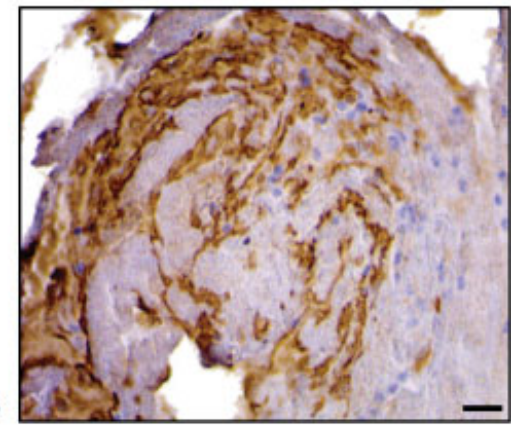

NE

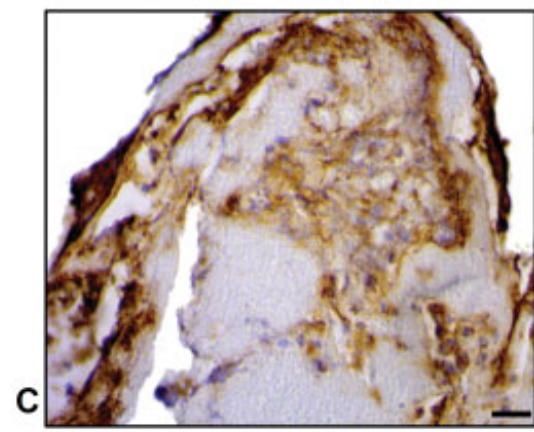

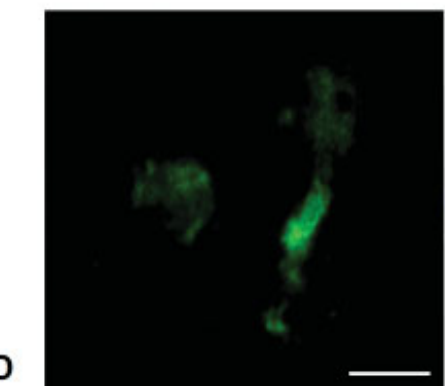
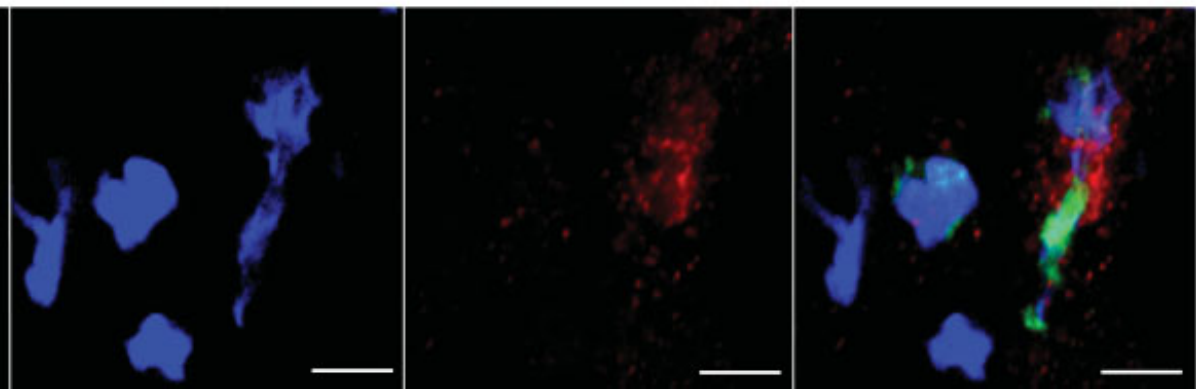

Fig. 2 NETs in stroke thrombi. Thrombi retrieved from stroke patients were analyzed via histology. The presence of citrullinated histones was analyzed via immunohistochemistry using an antibody against citrullinated histone $\mathrm{H} 3$ (H3Cit). (A) Zones of nuclear material that appeared extracellular were often observed on hematoxylin and eosin (H\&E) staining (arrowheads). (B, C) These chromatin strands corresponded with

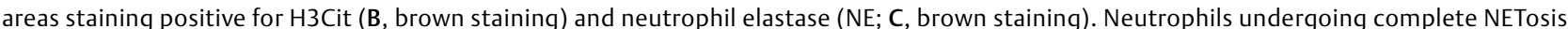
were also identified via triple costaining of H3Cit (green), the granulocyte marker CD66b (red), and DNA (DAPI, blue), as shown in panel D. Scale bars: $10 \mu \mathrm{m}$ in panels $\mathbf{A}, \mathbf{B}$, and $\mathbf{C}$ and $5 \mu \mathrm{m}$ in panel $\mathbf{D}$. (Adapted and reproduced with permission from Laridan et al. ${ }^{30}$ )

with noncardioembolic thrombi. ${ }^{30}$ The reason for such differences remains unclear, but the local environment (e.g., stasis vs. high shear) and thrombus maturation stage may in part influence the presence of NETs in the retrieved thrombi. We indeed found a higher presence of NETs in more mature, older stroke thrombi compared with younger, fresh thrombi. Ducroux et al recently performed histological analysis on 34 human ischemic stroke thrombi and similarly found that NETs were abundantly present in all thrombi via triple costaining positive for DNA, MPO, and citrullinated histone $\mathrm{H}_{4}{ }^{34}$ Interestingly, the presence of NETs was positively correlated with the length of thrombectomy procedure and number of device passes performed to achieve successful recanalization. Hence, NETs may participate in the interaction between thrombus and arterial wall, or between thrombus and thrombectomy device, further highlighting the potential clinical importance of NETs in thrombosis.

Besides myocardial infarction and ischemic stroke, NETs have also been found in other arterial pathological conditions, in particular in intraluminal thrombi derived from patients with abdominal aortic aneurysm (AAA). ${ }^{35,36}$ Detection of DNA-histone complexes was lower in older, more mature AAA thrombi (detected via computed tomography at least 1 year before retrieval), corresponding to reduced overall cellularity and higher collagen detection. Again, this suggests that NETs may play a role during thrombus formation and organization, which in later phases evolves to tissue remodeling. The differentiation of fibroblasts to collagen-producing myofibroblasts was induced by exposure to NETs in vitro, ${ }^{37}$ but the direct contribution of NETs in the context of arterial thrombosis has not yet been investigated. Importantly, infections can accelerate neutrophil recruitment and activation, leading to a more pronounced involvement of NETs in thrombosis. This was, for example, shown in patients with AAA, where Porphyromonas gingivalis infection (involved in chronic periodontitis) promoted neutrophil recruitment and NET formation and inhibited healing. ${ }^{35}$ Infection-driven thrombosis also led to NETs' detection of intraluminal lung thrombi in patients with acute interstitial pneumonitis $^{25}$ and sepsis. ${ }^{38}$

\section{Neutrophil Extracellular Traps in Venous Thrombi}

Venous thromboembolism comprises DVT, blood clots in large veins, and pulmonary embolism (PE), blood clots in pulmonary arteries likely originating as embolization of a venous thrombus. Animal models provided the first insight into the presence of NETs in venous thrombi. Histological analysis of a thrombus from an iliac vein balloon catheterization model in baboons revealed an extracellular chromatin core. Over the course of thrombus development, cell-free DNA in plasma became elevated. ${ }^{13}$ This was also seen in the inferior vena cava DVT mouse model induced by flow restriction in mice, ${ }^{18,39}$ along with the identification of citrullinated histones. ${ }^{39}$ These findings provided a basis for examining NETs in human VTE patients. 
A case report of a patient with microscopic polyangiitis (MPA) complicated with VTE identified NETs by immunostaining for citrullinated histones in both the affected kidney and also a deep vein thrombus at autopsy. ${ }^{40} \mathrm{~A}$ quantitative comparison of this thrombus with another patient's postoperative PE specimen showed that it contained a higher amount of NETs, which raised the possibility that certain venous thrombi can contain more NETs than others. As discussed, this could be due to the difference in etiology of the thrombus, here an autoimmune vasculitis, or also potentially due to the thrombus age or organization stage. It is often difficult to assess the true age of a thrombus prior to the appearance of symptoms, but histological examination can provide insight regarding the organization stage(s) of the thrombus. ${ }^{41}$ This was the basis for the study of Savchenko et al, where 16 VTE thrombi originating from 11 patients with DVT, PE, and/or an inferior vena cava filter thrombus were analyzed. ${ }^{42}$ After scoring the organization stage based on the Masson trichrome staining, consecutive sections were analyzed for the presence of neutrophils and citrullinated histone $\mathrm{H} 3$ (H3Cit) as a marker of extracellular NET structures or NETting neutrophils. Interestingly, NETs were predominantly found in the organizing parts of the thrombus and were much less present in already organized thrombi regions. This indicates that NET formation is likely transient, taking place with recruitment of neutrophils into the thrombus, and that as the thrombus matures, extracellular NETs are degraded and replaced with a collagen network. After thrombus formation, thrombus maturation into a tissue-like structure takes place over the following weeks, finally containing primarily collagen, fibroblasts, microvessels, and few leukocytes or platelets. As NETs can stimulate fibrotic remodeling, ${ }^{37}$ this provides another mechanism by which NETs could contribute to venous thrombus pathogenesis, by promoting development of a stable thrombus which is difficult to resolve with the body's endogenous thrombolytic pathways. This is of particularly relevance for the sequelae of VTE, such as postthrombotic syndrome or chronic thromboembolic pulmonary hypertension, and thus is of great interest for future investigation. Furthermore, as NETs could impact thrombus stability, a better understanding of thrombus composition could allow us to better predict the risk for DVT embolization. The effect of degree of NETs within a thrombus on embolization has not yet been investigated, and is worthy of study.

The relative contribution of NETs in venous versus arterial thrombosis is still unknown. Mangold et al examined NETs by immunostaining for DNA-histone complexes and performed a side-by-side comparison of 30 coronary artery thrombi and 7 deep vein thrombi, in both cases excised by thrombectomy. ${ }^{29}$ Based on this analysis, it was proposed that arterial thrombi could contain more NETs than venous thrombi. However, it is clear that there is a great heterogeneity in the content of NETs among different patients, ${ }^{30,42}$ and thus a study involving a larger number of venous thrombi is needed to fully address this question.

\section{Circulating Biomarkers of NETs and Thrombosis}

The concept of NETs contributing to thrombosis is supported by an increasing number of studies showing circulating biomarkers of NETosis in thrombotic pathologies. Cell-free DNA, MPO-DNA complexes, nucleosomes, and circulating citrullinated histones are indeed increased in various conditions, including sepsis, ${ }^{43-45}$ small vessel vasculitis, ${ }^{46}$ venous thrombosis, ${ }^{47-49}$ coronary atherosclerosis, ${ }^{50}$ and stroke. ${ }^{51-55}$ Interestingly, plasma levels of NETs markers often correlate with disease severity, as was shown in ischemic stroke ${ }^{51-53,56}$ and VTE. ${ }^{57}$ However, since many of these markers, with the exception of citrullinated histones, are not specific for NETosis and may also increase during general tissue damage and necrosis, suitable assays for specifically measuring circulating NET components or NET degradation products would be of high interest for future studies. Although NET biomarkers have been measured in venous and arterial thrombosis patients separately, a study comparing the two has not yet been performed. Measuring NET biomarkers may be of broader clinical relevance, such as in predicting the progression to sepsis in burn patients. ${ }^{58}$ As different types of bacteria induce varying amounts of NET release $^{59}$ and cell-free DNA strongly correlates with prognosis in severe sepsis, ${ }^{60}$ comparing NET biomarkers across different infectious sources in sepsis linked to outcome could be valuable.

\section{Neutrophil Extracellular Traps: A New Therapeutic Target?}

The involvement of NETs in both arterial and venous thrombosis creates the interesting possibility to develop novel therapeutic strategies targeting NETs, to either reduce thrombosis or improve thrombolysis. As a scaffold for thrombus formation, NETs can contribute to overall thrombus stability, conferring resistance to thrombolysis. Hence, degradation of NETs structure, in addition to fibrin breakdown via the fibrinolytic system, could become a promising thrombolytic option. NETs are composed of decondensed chromatin networks and are thus vulnerable to nuclease activity. Certain nuclease-producing microbes use such nucleases to evade the toxic effect of NETs. ${ }^{61,62}$ In addition, host DNases, such as DNase1 and DNase1-like 3, were recently shown to counteract the deleterious effects of intravascular NET formation. ${ }^{38}$ DNase 1 is the predominant nuclease in plasma. After finding the presence of NETs in thrombi from ischemic stroke patients, we tested whether pharmacological breakdown of NETs using DNase1 could enhance thrombus dissolution. ${ }^{30}$ Although treatment of stroke thrombi with tissue plasminogen activator ( $t$-PA) alone induced gradual, partial lysis of the thrombi, addition of DNase1 significantly accelerated ex vivo lysis. ${ }^{30}$ These findings showed that DNase1 can promote ex vivo stroke thrombus dissolution, providing proof of concept to target NETs as a novel prothrombolytic strategy in ischemic stroke. Similar results were recently reported by Ducroux et al, who 
in addition showed that treatment with DNase 1 alone had no thrombolytic effect on ischemic stroke thrombi. ${ }^{34}$ Indeed, combination of both fibrinolysis and nuclease activity is needed to induce successful thrombolysis. When blood clots were generated in vitro in the presence of activated neutrophils releasing NETs, treatment with t-PA alone was effective to degrade most of the fibrin, but resulted in clots that were still held together by a scaffold of extracellular DNA. ${ }^{13}$ Furthermore, DNA and histones were shown to modify the structure of fibrin into thicker fibrin fibers, thus resulting in increased resistance to mechanical and enzymatic destruction. ${ }^{63}$ Interestingly, DNase 1 activity may benefit from the plasminogen system, as plasmin can degrade histones, thereby facilitating chromatin degradation by DNase $1 .{ }^{64}$

Addition of DNase 1 also accelerated ex vivo tPA-mediated thrombus lysis on coronary thrombi retrieved from patients with acute coronary syndrome, ${ }^{29}$ thus suggesting a broad therapeutic potential of DNase1. Importantly, DNase1 already is a safe, low-cost, U.S. Food and Drug Administration (FDA)-approved drug routinely used for cystic fibrosis to clear extracellular DNA in the lungs. Combination of DNase1 with t-PA could potentially allow decreasing the dose of t-PA utilized, so limiting its side effects and potentially increasing its therapeutic time window. In particular, DNase1 could be used in combination with the VWF-cleaving enzyme ADAMTS13, of which we previously demonstrated thrombolytic activity, able to dissolve tPA-resistant cerebral occlusion in a mouse model of ischemic stroke. ${ }^{65}$

In addition to digesting NETs, another potential therapeutic approach is neutralizing their harmful components. Heparin has been shown to dismantle NETs and to neutralize to harmful effects of histones, probably due to electrostatic interactions between negatively charged heparin and histones. $^{13,15}$ APC cleaves histones, thereby reducing their cytotoxicity, ${ }^{14}$ an effect that can be potentiated via thrombomodulin alfa-mediated increase of APC generation. ${ }^{66}$ To circumvent the potential bleeding risk associated with the anticoagulant properties of heparin and APC, non-anticoagulant variants could become of high interest. ${ }^{67-69}$

Recurrent thrombosis is a concern in both arterial and venous thrombosis. In patients who have previously experienced a thrombotic event, prevention of NET formation could be beneficial. Inhibition of PAD4 may become a promising strategy, as PAD4 deficiency reduced thrombotic occurrence in a mouse model of venous thrombosis. ${ }^{6}$ Importantly, PAD4 deficiency did not lead to increased susceptibility to bacterial infection in animal models, ${ }^{70,71}$ indicating that this approach would not likely lead to a detrimental immunosuppressive effect due to a lack of NETs. Selective PAD4 inhibitors that prevent NET formation have been developed, and further research is needed to validate their therapeutic potential. ${ }^{72}$ Also, pharmacological inhibition of Janus kinase (JAK) signaling has been proposed to reduce NETosis and thrombosis. ${ }^{73}$ Ruxolitinib, an FDA-approved inhibitor of JAK, abrogated NET formation and reduced thrombosis in a mouse model of deep vein stenosis. ${ }^{73}$ Although the exact mechanisms by which the JAK pathway interferes with NET formation is not still completely clear, regulation of PAD4 is one hypothesis. While patients receiving ruxolitinib had a lower risk of VTE events in the RESPONSE trial, ${ }^{74}$ more studies, specifically designed to evaluate NETosis and its link with thrombotic risk in ruxolitinib-treated patients, would be of interest in the future.

\section{Conclusion}

Neutrophil extracellular traps have become undeniable actors in the field of thrombosis and hemostasis. NETs regulate thrombosis in different ways and are implicated in the pathophysiology of both arterial and venous thrombotic complications. Without a doubt, future studies will further fine-tune our knowledge on temporal and spatial processes of neutrophil-driven thrombus formation and maturation. This information will become highly valuable for developing novel antithrombotic therapies. Pharmacological disassembly and degradation of NETs in thrombi can enhance acute thrombolysis. In addition, preventing the formation of NETs can reduce thrombogenicity, which might become beneficial in thrombosis prevention. Preclinical and clinical studies investigating these new therapeutic opportunities are now needed to fully understand the efficacy and safety of targeting NETs in thrombosis.

\section{Conflicts of Interest}

E.L. has no conflict of interest to disclose. S.F.D.M. and K.M. are inventors on the granted patent US9642822 covering the targeting of NETs in thrombosis. K.M. is an inventor on U.S. patent application $62 / 594,266$ that covers the use of ruxolitinib and inhibition of JAK-STAT signaling to inhibit the formation of NETs.

\section{Acknowledgments}

This work was supported by the Fonds voor Wetenschappelijk Onderzoek - Vlaanderen (Strategic Basic Research Doctoral Grant 1S25216N to E.L. and research grants G.0A86.13, G.0785.17, and $1509216 \mathrm{~N}$ to S.F.D.M), by an "Onderzoekstoelage" grant from KU Leuven (OT/14/099 to S.F.D.M) and by a research grant from the Queen Elisabeth Medical Foundation (to S.F.D.M). K.M. is the recipient of a fellowship from the European Union's Horizon 2020 research and innovation program under the Marie Skłodowska-Curie Grant Agreement No. 747993.

\section{References}

1 Lozano R, Naghavi M, Foreman K, et al. Global and regional mortality from 235 causes of death for 20 age groups in 1990 and 2010: a systematic analysis for the Global Burden of Disease Study 2010. Lancet 2012;380(9859):2095-2128

2 Martinod K, Wagner DD. Thrombosis: tangled up in NETs. Blood 2014;123(18):2768-2776

3 Brinkmann V, Reichard U, Goosmann C, et al. Neutrophil extracellular traps kill bacteria. Science 2004;303(5663):1532-1535

4 Zawrotniak M, Rapala-Kozik M. Neutrophil extracellular traps (NETs) - formation and implications. Acta Biochim Pol 2013;60 (03):277-284 
5 Fuchs TA, Abed U, Goosmann C, et al. Novel cell death program leads to neutrophil extracellular traps. J Cell Biol 2007;176(02): 231-241

6 Martinod K, Demers M, Fuchs TA, et al. Neutrophil histone modification by peptidylarginine deiminase 4 is critical for deep vein thrombosis in mice. Proc Natl Acad Sci U S A 2013; 110(21):8674-8679

7 Neeli I, Khan SN, Radic M. Histone deimination as a response to inflammatory stimuli in neutrophils. J Immunol 2008;180(03): 1895-1902

8 Papayannopoulos V, Metzler KD, Hakkim A, Zychlinsky A Neutrophil elastase and myeloperoxidase regulate the formation of neutrophil extracellular traps. J Cell Biol 2010;191(03): 677-691

9 Yipp BG, Kubes P. NETosis: how vital is it? Blood 2013;122(16): 2784-2794

10 Massberg S, Grahl L, von Bruehl M-L, et al. Reciprocal coupling of coagulation and innate immunity via neutrophil serine proteases. Nat Med 2010;16(08):887-896

11 McDonald B, Urrutia R, Yipp BG, Jenne CN, Kubes P. Intravascular neutrophil extracellular traps capture bacteria from the bloodstream during sepsis. Cell Host Microbe 2012;12(03):324-333

12 Pfeiler S, Stark K, Massberg S, Engelmann B. Propagation of thrombosis by neutrophils and extracellular nucleosome networks. Haematologica 2017;102(02):206-213

13 Fuchs TA, Brill A, Duerschmied D, et al. Extracellular DNA traps promote thrombosis. Proc Natl Acad Sci U S A 2010;107(36): 15880-15885

$14 \mathrm{Xu}$ J, Zhang X, Pelayo R, et al. Extracellular histones are major mediators of death in sepsis. Nat Med 2009;15(11):1318-1321

15 Fuchs TA, Bhandari AA, Wagner DD. Histones induce rapid and profound thrombocytopenia in mice. Blood 2011;118(13): 3708-3714

16 Semeraro F, Ammollo CT, Morrissey JH, et al. Extracellular histones promote thrombin generation through platelet-dependent mechanisms: involvement of platelet TLR2 and TLR4. Blood 2011; 118(07):1952-1961

17 Ammollo CT, Semeraro F, Xu J, Esmon NL, Esmon CT. Extracellular histones increase plasma thrombin generation by impairing thrombomodulin-dependent protein $\mathrm{C}$ activation. J Thromb Haemost 2011;9(09):1795-1803

18 von Brühl M-L, Stark K, Steinhart A, et al. Monocytes, neutrophils, and platelets cooperate to initiate and propagate venous thrombosis in mice in vivo. J Exp Med 2012;209(04):819-835

19 Noubouossie DF, Whelihan MF, Yu Y-B, et al. In vitro activation of coagulation by human neutrophil DNA and histone proteins but not neutrophil extracellular traps. Blood 2017;129(08): 1021-1029

20 Gould TJ, Vu TT, Swystun LL, et al. Neutrophil extracellular traps promote thrombin generation through platelet-dependent and platelet-independent mechanisms. Arterioscler Thromb Vasc Biol 2014;34(09):1977-1984

21 Etulain J, Martinod K, Wong SL, Cifuni SM, Schattner M, Wagner DD. P-selectin promotes neutrophil extracellular trap formation in mice. Blood 2015;126(02):242-246

22 Maugeri N, Campana L, Gavina M, et al. Activated platelets present high mobility group box 1 to neutrophils, inducing autophagy and promoting the extrusion of neutrophil extracellular traps. J Thromb Haemost 2014;12(12):2074-2088

23 Stark K, Philippi V, Stockhausen S, et al. Disulfide HMGB1 derived from platelets coordinates venous thrombosis in mice. Blood 2016;128(20):2435-2449

24 Dyer MR, Chen Q, Haldeman S, et al. Deep vein thrombosis in mice is regulated by platelet HMGB1 through release of neutrophilextracellular traps and DNA. Sci Rep 2018;8(01):2068

25 Clark SR, Ma AC, Tavener SA, et al. Platelet TLR4 activates neutrophil extracellular traps to ensnare bacteria in septic blood. Nat Med 2007;13(04):463-469
26 Naruko T, Ueda M, Haze K, et al. Neutrophil infiltration of culprit lesions in acute coronary syndromes. Circulation 2002;106(23): 2894-2900

27 de Boer OJ, Li X, Teeling P, et al. Neutrophils, neutrophil extracellular traps and interleukin-17 associate with the organisation of thrombi in acute myocardial infarction. Thromb Haemost 2013;109(02):290-297

28 Riegger J, Byrne RA, Joner M, et al; Prevention of Late Stent Thrombosis by an Interdisciplinary Global European Effort (PRESTIGE) Investigators. Histopathological evaluation of thrombus in patients presenting with stent thrombosis. A multicenter European study: a report of the prevention of late stent thrombosis by an interdisciplinary global European effort consortium. Eur Heart J 2016;37(19):1538-1549

29 Mangold A, Alias S, Scherz T, et al. Coronary neutrophil extracellular trap burden and deoxyribonuclease activity in ST-elevation acute coronary syndrome are predictors of ST-segment resolution and infarct size. Circ Res 2015;116(07):1182-1192

30 Laridan E, Denorme F, Desender L, et al. Neutrophil extracellular traps in ischemic stroke thrombi. Ann Neurol 2017;82(02): 223-232

31 De Meyer SF, Andersson T, Baxter B, et al; Clot Summit Group. Analyses of thrombi in acute ischemic stroke: a consensus statement on current knowledge and future directions. Int J Stroke 2017;12(06):606-614

32 Boeckh-Behrens T, Schubert M, Förschler A, et al. The impact of histological clot composition in embolic stroke. Clin Neuroradiol 2016;26(02):189-197

33 Boeckh-Behrens T, Kleine JF, Zimmer C, et al. Thrombus histology suggests cardioembolic cause in cryptogenic stroke. Stroke 2016; 47(07):1864-1871

34 Ducroux C, Di Meglio L, Loyau S, et al. Thrombus neutrophil extracellular traps content impair tPA-induced thrombolysis in acute ischemic stroke. Stroke 2018;49(03):754-757

35 Delbosc S, Alsac J-M, Journe C, et al. Porphyromonas gingivalis participates in pathogenesis of human abdominal aortic aneurysm by neutrophil activation. Proof of concept in rats. PLoS One 2011;6(04):e18679

36 Oklu R, Albadawi H, Watkins MT, Monestier M, Sillesen M, Wicky S. Detection of extracellular genomic DNA scaffold in human thrombus: implications for the use of deoxyribonuclease enzymes in thrombolysis. J Vasc Interv Radiol 2012;23(05): $712-718$

37 Chrysanthopoulou A, Mitroulis I, Apostolidou E, et al. Neutrophil extracellular traps promote differentiation and function of fibroblasts. J Pathol 2014;233(03):294-307

38 Jiménez-Alcázar M, Rangaswamy C, Panda R, et al. Host DNases prevent vascular occlusion by neutrophil extracellular traps. Science 2017;358(6367):1202-1206

39 Brill A, Fuchs TA, Savchenko AS, et al. Neutrophil extracellular traps promote deep vein thrombosis in mice. J Thromb Haemost 2012;10(01):136-144

40 Nakazawa D, Tomaru U, Yamamoto C, Jodo S, Ishizu A. Abundant neutrophil extracellular traps in thrombus of patient with microscopic polyangiitis. Front Immunol 2012;3:333

41 Seidman MA, Mitchell RN. Surgical pathology of small- and medium-sized vessels. Surg Pathol Clin 2012;5(02):435-451

42 Savchenko AS, Martinod K, Seidman MA, et al. Neutrophil extracellular traps form predominantly during the organizing stage of human venous thromboembolism development. J Thromb Haemost 2014;12(06):860-870

43 Margraf S, Lögters T, Reipen J, Altrichter J, Scholz M, Windolf J. Neutrophil-derived circulating free DNA (cf-DNA/NETs): a potential prognostic marker for posttraumatic development of inflammatory second hit and sepsis. Shock 2008;30(04):352-358

44 Martins GA, Kawamura MT, Carvalho MdaG. Detection of DNA in the plasma of septic patients. Ann N Y Acad Sci 2000;906(01): $134-140$ 
45 Zeerleder S, Zwart B, Wuillemin WA, et al. Elevated nucleosome levels in systemic inflammation and sepsis. Crit Care Med 2003, 31(07):1947-1951

46 Kessenbrock K, Krumbholz M, Schönermarck U, et al. Netting neutrophils in autoimmune small-vessel vasculitis. Nat Med 2009;15(06):623-625

47 Stassen PM, Derks RPH, Kallenberg CGM, Stegeman CA. Venous thromboembolism in ANCA-associated vasculitis-incidence and risk factors. Rheumatology (Oxford) 2008;47(04):530-534

48 van Montfoort ML, Stephan F, Lauw MN, et al. Circulating nucleosomes and neutrophil activation as risk factors for deep vein thrombosis. Arterioscler Thromb Vasc Biol 2013;33(01): 147-151

49 Diaz JA, Fuchs TA, Jackson TO, et al; for the Michigan Research Venous Group*. Plasma DNA is elevated in patients with deep vein thrombosis. J Vasc Surg Venous Lymphat Disord 2013;1(04): 341-348

50 Borissoff JI, Joosen IA, Versteylen MO, et al. Elevated levels of circulating DNA and chromatin are independently associated with severe coronary atherosclerosis and a prothrombotic state. Arterioscler Thromb Vasc Biol 2013;33(08):2032-2040

51 Tsai N-W, Lin T-K, Chen S-D, et al. The value of serial plasma nuclear and mitochondrial DNA levels in patients with acute ischemic stroke. Clin Chim Acta 2011;412(5-6):476-479

52 Geiger S, Holdenrieder S, Stieber P, et al. Nucleosomes in serum of patients with early cerebral stroke. Cerebrovasc Dis 2006;21(12):32-37

53 Rainer TH, Wong LKS, Lam W, et al. Prognostic use of circulating plasma nucleic acid concentrations in patients with acute stroke. Clin Chem 2003;49(04):562-569

54 Thålin C, Demers M, Blomgren B, et al. NETosis promotes cancerassociated arterial microthrombosis presenting as ischemic stroke with troponin elevation. Thromb Res 2016;139:56-64

55 Hirose T, Hamaguchi S, Matsumoto N, et al. Presence of neutrophil extracellular traps and citrullinated histone $\mathrm{H} 3$ in the bloodstream of critically ill patients. PLoS One 2014;9(11):e111755

56 Vallés J, Lago A, Santos MT, et al. Neutrophil extracellular traps are increased in patients with acute ischemic stroke: prognostic significance. Thromb Haemost 2017;117(10):1919-1929

57 Mauracher L-M, Posch F, Martinod K, et al. Citrullinated histone H3, a biomarker of neutrophil extracellular trap formation, predicts the risk of venous thromboembolism in cancer patients. J Thromb Haemost 2018;16(03):508-518

58 Hampson P, Dinsdale RJ, Wearn CM, et al. Neutrophil dysfunction, immature granulocytes, and cell-free DNA are early biomarkers of sepsis in burn-injured patients: a prospective observational cohort study. Ann Surg 2017;265(06):1241-1249

59 Van Ziffle JA, Lowell CA. Neutrophil-specific deletion of Syk kinase results in reduced host defense to bacterial infection. Blood 2009, 114(23):4871-4882
60 Dwivedi DJ, Toltl LJ, Swystun LL, et al; Canadian Critical Care Translational Biology Group. Prognostic utility and characterization of cell-free DNA in patients with severe sepsis. Crit Care 2012;16(04):R151

61 Beiter T, Fragasso A, Hartl D, Nieß AM. Neutrophil extracellular traps: a walk on the wild side of exercise immunology. Sports Med 2015;45(05):625-640

62 Buchanan JT, Simpson AJ, Aziz RK, et al. DNase expression allows the pathogen group A Streptococcus to escape killing in neutrophil extracellular traps. Curr Biol 2006;16(04):396-400

63 Longstaff C, Varjú I, Sótonyi P, et al. Mechanical stability and fibrinolytic resistance of clots containing fibrin, DNA, and histones. J Biol Chem 2013;288(10):6946-6956

64 Napirei M, Wulf S, Mannherz HG. Chromatin breakdown during necrosis by serum Dnase 1 and the plasminogen system. Arthritis Rheum 2004;50(06):1873-1883

65 Wyseure T, Rubio M, Denorme F, et al. Innovative thrombolytic strategy using a heterodimer diabody against TAFI and PAI-1 in mouse models of thrombosis and stroke. Blood 2015;125(08): 1325-1332

66 Osada K, Minami T, Arioka T, et al. Thrombomodulin alfa attenuates the procoagulant effect and cytotoxicity of extracellular histones through the promotion of protein $\mathrm{C}$ activation. Thromb Res 2017;160:51-57

67 Andreou AP, Efthymiou M, Yu Y, et al. Protective effects of nonanticoagulant activated protein $\mathrm{C}$ variant (D36A/L38D/A39V) in a murine model of ischaemic stroke. PLoS One 2015;10(04):e0122410

68 Wildhagen KC, García de Frutos P, Reutelingsperger CP, et al. Nonanticoagulant heparin prevents histone-mediated cytotoxicity in vitro and improves survival in sepsis. Blood 2014;123(07): 1098-1101

69 Wang Y, Thiyagarajan M, Chow N, et al. Differential neuroprotection and risk for bleeding from activated protein $\mathrm{C}$ with varying degrees of anticoagulant activity. Stroke 2009;40(05): 1864-1869

70 Martinod K, Fuchs TA, Zitomersky NL, et al. PAD4-deficiency does not affect bacteremia in polymicrobial sepsis and ameliorates endotoxemic shock. Blood 2015;125(12):1948-1956

71 Kolaczkowska E, Jenne CN, Surewaard BGJ, et al. Molecular mechanisms of NET formation and degradation revealed by intravital imaging in the liver vasculature. Nat Commun 2015;6:6673

72 Lewis HD, Liddle J, Coote JE, et al. Inhibition of PAD4 activity is sufficient to disrupt mouse and human NET formation. Nat Chem Biol 2015;11(03):189-191

73 Wolach O, Sellar RS, Martinod K, et al. Increased neutrophil extracellular trap formation promotes thrombosis in myeloproliferative neoplasms. Sci Transl Med 2018;10(436):8292

74 Vannucchi AM, Kiladjian JJ, Griesshammer M, et al. Ruxolitinib versus standard therapy for the treatment of polycythemia vera. $\mathrm{N}$ Engl J Med 2015;372(05):426-435 